\title{
REVIEW \\ Development of Typing Methods of Actinobacillus pleuropneumoniae Based on the Antigenic and Genetic Diversity of the Protective Outer Membrane Lipoprotein
}

\author{
Hiroya ITO ${ }^{1 *}$ \\ Kyushu Research Station, Research Team for Environmental and Enzootic Diseases, National \\ Institute of Animal Health, National Agriculture and Food Research Organization (NARO) \\ (Kagoshima, Kagoshima 891-0105, Japan)
}

\begin{abstract}
Actinobacillus pleuropneumoniae is a causative agent of porcine pleuropneumonia that is one of the important bacterial diseases of the respiratory tract of pigs. The disease occurs worldwide and results in serious economic losses to the pig-rearing industry. In this review, new typing methods based on the antigenic and genetic diversity of a protective outer membrane lipoprotein (OmlA), that have been developed for control and epidemiological study of the disease, are described. First, the gene for the OmlA of an A. pleuropneumoniae field isolate (serotype 5a) has been cloned and characterized. The lipoprotein has been shown to be a protective antigen of the organism. Southern blot and the nucleotide sequence analysis of the gene for the lipoprotein have revealed the presence and diversity of the gene in A. pleuropneumoniae. Secondly, based on the antigenic diversity of OmlA, Western blot analysis with specific antisera against three antigenically distinct OmlAs has been developed. The antisera can differentiate A. pleuropneumoniae into three groups. Finally, based on the genetic diversity of OmlA, a PCR-Restriction Fragment Length Polymorphism (RFLP) typing method has been developed. This method can divide A. pleuropneumoniae into five groups. The grouping results by the Western blot analysis were correlated with that by the PCR-RFLP typing method. In addition, the PCR-RFLP typing method can be performed in almost all veterinary diagnostic laboratories since the method does not require specific antisera and expensive reagents and apparatus. Therefore, the PCR-RFLP typing method can be substituted for other OmlA typing and seems to be the most convenient and practical OmlA typing method at present. The new typing methods developed in this study should be used as tools which are effective and practical for control of porcine pleuropneumonia caused by A. pleuropneumoniae.
\end{abstract}

Discipline: Animal Health

Additional key words: PCR-RFLP, Porcine pleuropneumonia, Western blot analysis

\section{Introduction}

Actinobacillus pleuropneumoniae is a causative agent of porcine pleuropneumonia that is characterized by a fibrinous-hemorrhagic pneumonia and is one of the important bacterial diseases of the respiratory tract of pigs $^{36}$. To date, 15 serotypes are recognized based on an- tigenic diversity of capsular polysaccharide and lipopolysaccharide ${ }^{3,31}$. The disease occurs worldwide and results in serious economic losses to the pig-rearing industry ${ }^{36}$. Prevalent serotypes differ according to country ${ }^{1,3,7,10,29,37,39}$, area and farm.

At present polyclonal antisera raised against whole bacterial cells of A. pleuropneumoniae are utilized for serotyping ${ }^{20,21,26,27}$. However, only a few veterinary diag-

\footnotetext{
Present address:

${ }^{1}$ Center for Animal Disease Control and Protection / Research Team for Environmental and Enzootic Diseases, National Institute of Animal Health, NARO (Tsukuba, Ibaraki 305-0035, Japan)

*Corresponding author: e-mail itohiroy@affrc.go.jp

Received 17 August 2007; accepted 10 April 2008.
} 
nostic laboratories can prepare a full set of serotype-specific antisera for serotyping. Furthermore, cross-reactions are often observed among different serotypes in a number of the methods, which prevent accurate typing $12,22,23,24,25,28$. The cross-reactions are most likely due to the fact that the polyclonal antisera contain antibodies directed against a variety of antigens including antigens common in A. pleuropneumoniae. In addition, quantitative differences of expressed antigens including both serotype-specific and cross-reacting antigens may affect the results of typing. For these reasons, the development of a new typing method that is not affected by cross-reacting antigens and is useful for prevention of porcine pleuropneumonia is desirable. Therefore, it seems that development of a new typing method based on a particular single antigen and gene would facilitate a more precise typing of A. pleuropneumoniae isolates without influence from the cross-reacting antigens.

Furthermore, if the antigen for typing were a protective antigen of $A$. pleuropneumoniae, the new typing method could be used as a tool which is directly effective and practical for control and epidemiological analysis of the disease. Especially, to know the antigenic type of protective antigens is important for the appropriate control of porcine pleuropneumonia by vaccination.

This review is part of a series of studies conducted to develop a new typing method based on a particular protective antigen (an outer membrane lipoprotein (OmlA protein)) and its gene (omlA gene) of A. pleuropneumoni$a$. The usefulness and importance of the OmlA protein for the prevention of $A$. pleuropneumoniae infection ${ }^{4,11,16}$ are also manifest by the fact that a recombinant vaccine that contains the OmlA protein as one of protective components is licensed by a vaccine company in North America $^{2}$. The usefulness and importance of the omlA gene for diagnosis of $A$. pleuropneumoniae infection are also proved by other researchers' reports,8,13,14,32,33. Currently a diagnostic kit for detection and typing of A. pleuropneumoniae using the omlA-PCR is marketed by a company in France $^{8,32}$. Species specificity of a number of the omlAPCR systems has also been examined, and they have been shown to be species-specific ${ }^{13,32,33,38}$.

\section{Cloning and characterization of an outer membrane lipoprotein of an Actinobacillus pleuropneumoniae serotype 5 a field isolate ${ }^{16}$}

The gene encoding an OmlA protein with a molecular mass of 43-kDa was cloned from a Japanese field isolate of Actinobacillus pleuropneumoniae (strain NG-8, serotype $5 a)^{16}$. Immunization of the OmlA protein protected mice from death in a challenge experiment with an $\begin{array}{llllllllllll}1 & 2 & 3 & 4 & 5 a & 5 b & 6 & 7 & 8 & 9 & 10 & 11\end{array}$

A

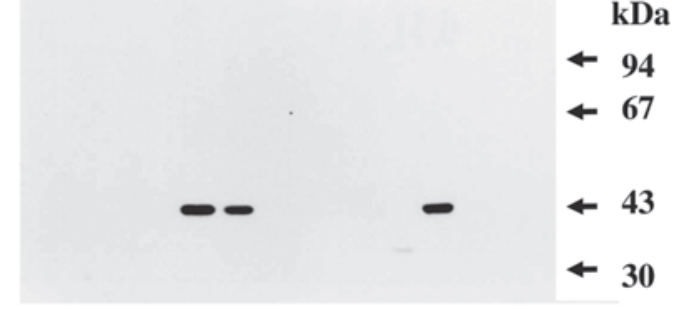

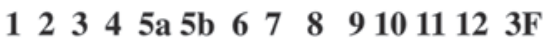

B
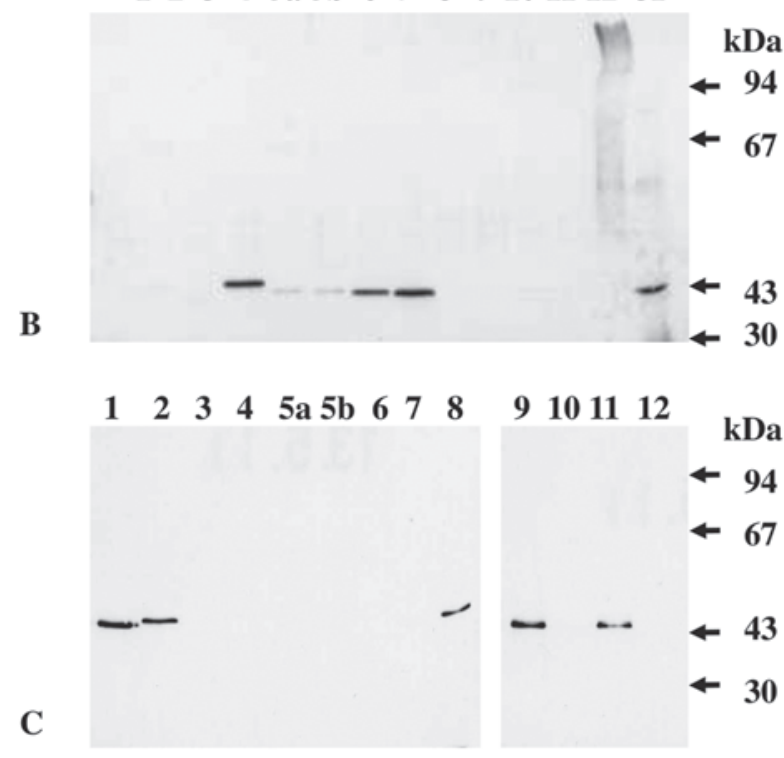

Fig. 1. Western blot of whole-cell lysates of A. pleuropneumoniae serotype reference and field strains

The top blot (A) was probed with the antiserum against the OmlA protein of an A. pleuropneumoniae field isolate (NG-8, serotype $5 \mathrm{a}$ ). The middle blot (B) was probed with the antiserum against the OmlA protein of an A. pleuropneumoniae reference strain (WF83, serotype 7). The bottom blot (C) was probed with the antiserum against the OmlA protein of an A. pleuropneumoniae reference strain (serotype 1). Numbers above each lane indicate the serotype of the reference strain. 3F indicates the field strain of serotype 3. Molecular mass markers are shown on the right (in $\mathrm{kDa}$ ).

A. pleuropneumoniae serotype 5 , indicating that OmlA protein is a protective antigen of A. pleuropneumoniae ${ }^{16}$. The protective efficacy of the OmlA protein from $A$. pleuropneumoniae serotype 5 has been supported by a challenge experiment with pigs by other researchers ${ }^{4}$. Southern blot analysis under low-stringency conditions revealed the presence of a hybridizing sequence in reference strains of serotypes 1, 2, 3, 4, 5a, 5b, 6, 7, 8, 9, 10, 11, and 12, which are prevalent serotypes in Japan 1,10,37,39 except for serotypes 4 and 10, indicating the presence of allelic variants of omlA gene in the strains of these sero- 
types $^{16}$. While, Southern blot analysis under high-stringency conditions showed the presence of a sequence highly homologous to the omlA gene of strain NG-8 in reference strains of serotypes $5 \mathrm{a}, 5 \mathrm{~b}$ and $10^{16}$. In addition, a specific serum against OmlA protein of NG-8 detected a homologous protein in the strains of serotypes $5 \mathrm{a}, 5 \mathrm{~b}$ and 10 (Fig. 1A), suggesting that these serotypes carry an omlA gene and an OmlA protein which are each highly similar among the serotypes. From the data obtained by Western (Fig. 1A) and Southern blot analysis ${ }^{16}$, the presence of antigenic and genetic diversity of an A. pleuropneumoniae OmlA protein was indicated. In conjunction with a protective efficacy of OmlA protein, general presence of omlA gene in A. pleuropneumoniae and its potential genetic diversity indicate that the OmlA protein and its gene can be used for typing of A. pleuropneumoniae which is directly effective and practical for appropriate control of porcine pleuropneumonia caused by A. pleuropneumoniae.

\section{Genetic diversity of the outer membrane lipoprotein}

Nucleotide sequence determination of DNA containing the omlA-like gene in reference strains of A. pleuropneumoniae serotype 1 to 12 (the accession number: AB007572 to AB007584, respectively) revealed that the omlA gene generally exists in A. pleuropneumoniae isolates. The genetic diversity of the omlA gene and the OmlA protein among A. pleuropneumoniae serotypes has been exhibited by the comparison of the gene (Table 1). The positions of the conserved and variable sequences of the omlA gene among $A$. pleuropneumoniae serotypes could be identified. The nucleotide sequence-based typing of the omlA genes could be used to differentiate $A$. pleuropneumoniae isolates since the phylogenetic analysis of the nucleotide sequence of omlA genes (Fig. 2 and Table 2) divided A. pleuropneumoniae serotypes into four groups: Group I, serotype 1, 9, 11, and 12; Group II, sero-

Table 1. Percentage identities in nucleotide sequence between the omlA gene of reference strains of A. pleuropneumoniae serotypes 1 to 12

\begin{tabular}{|c|c|c|c|c|}
\hline \multirow{2}{*}{ Gene } & \multicolumn{4}{|c|}{ \% pairwise identities in nucleotide sequences } \\
\hline & Group I & Group II & Group III & Group IV \\
\hline $\begin{array}{l}\text { Group I }^{\text {a) }} \\
\text { Serotype } 1,9 \text { and } 11\end{array}$ & 99.3 to 100 & 77.5 to 82.2 & 63.0 to 75.6 & 63.3 to 72.5 \\
\hline $\begin{array}{l}\text { Group } \mathrm{II}^{\mathrm{a})} \\
\text { Serotype } 2 \text { and } 8\end{array}$ & & 100 & 70.7 to 73.6 & 74.2 to 74.5 \\
\hline $\begin{array}{l}\text { Group III }^{\text {a) }} \\
\text { Serotype } 3,4,6 \text {, and } 7\end{array}$ & & & 95.4 to 99.9 & 69.5 to 79.1 \\
\hline $\begin{array}{l}\text { Group IV }{ }^{\text {a) }} \\
\text { Serotype 5a, 5b and } 10\end{array}$ & & & & 99.6 to 99.8 \\
\hline
\end{tabular}

Table 2. Grouping based on the antigenic and genetic diversity of the OmlA protein and the omlA gene

\begin{tabular}{|c|c|c|c|c|c|}
\hline \multicolumn{2}{|c|}{$\begin{array}{l}\text { Grouping by the } \\
\text { Western blot analysis }\end{array}$} & \multicolumn{2}{|c|}{$\begin{array}{l}\text { Grouping by the } \\
\text { nucleotide sequencing analysis }{ }^{\mathrm{b})}\end{array}$} & \multicolumn{2}{|c|}{$\begin{array}{c}\text { Grouping by the } \\
\text { PCR-RFLP analysis }\end{array}$} \\
\hline Antigenic group & Serotype & Sequence group & Serotype & PCR-RFLP group & Serotype \\
\hline \multirow{2}{*}{ A } & \multirow{2}{*}{$1,2,8,9$, and 11} & I & $1,9,11$, and 12 & I & $1,9,11$, and 12 \\
\hline & & II & 2 and 8 & II & 2 and 8 \\
\hline \multirow{2}{*}{ B } & \multirow{2}{*}{$3^{\mathrm{d})}, 4,6$, and 7} & \multirow{2}{*}{ III } & \multirow{2}{*}{$3,4,6$, and 7} & III & 3,6 and 7 \\
\hline & & & & IV & 4 \\
\hline $\mathrm{C}$ & 5a, 5b and 10 & IV & 5a, 5b and 10 & $\mathrm{~V}$ & 5a, 5b and 10 \\
\hline
\end{tabular}

a): Antigenic typing method developed in this study. Serotype 12 strain cannot be grouped into any group since it did not express any detectable OmlA protein reacting with the antisera prepared in this study.

b): Genetic typing method developed in this study.

c): Genetic typing method developed in this study.

d): A field isolate of A. pleuropneumoniae serotype 3 belongs to Group B (Fig. 1). While serotype 3 reference strain did not express any detectable OmlA protein reacting with the antisera prepared in this study. 


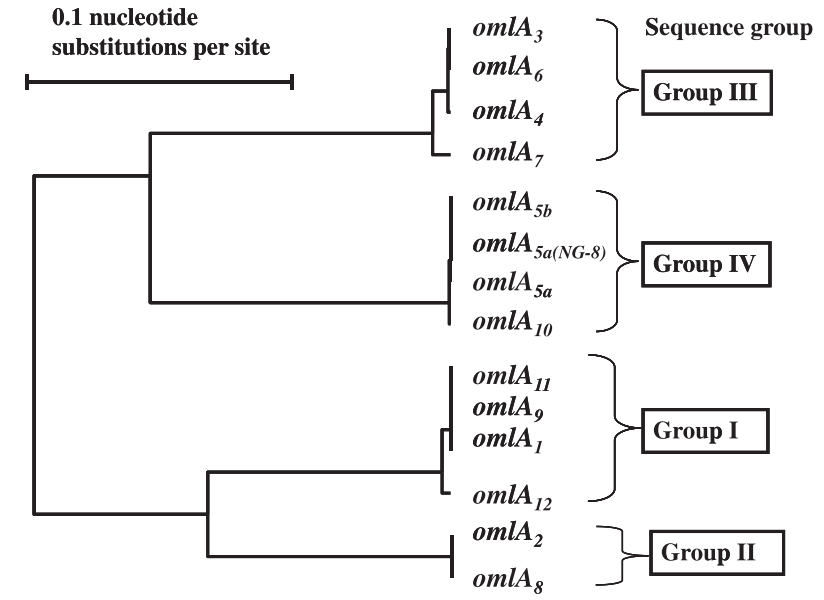

Fig. 2. Phylogenetic tree based on the nucleotide sequence conducted by UPGMA of Genetyx version 7

Number following "omlA" indicates the serotype.

type 2 and 8; Group III, serotype 3, 4, 6, and 7; and Group $\mathrm{IV}$, serotype $5 \mathrm{a}, 5 \mathrm{~b}$ and 10 . Since a DNA sequencer and reagents for nucleotide sequence determination are extremely expensive, only a very few veterinary diagnostic laboratories can determine the nucleotide sequence of the omlA gene by themselves. Therefore we have subsequently developed typing methods that are less expensive and can be performed without expensive apparatus in standard veterinary diagnostic laboratories such as Livestock Hygiene Centers in Japan.

\section{Development of typing method based on the antigenic diversity of the outer membrane lipoprotein $^{16,17}$}

A new serotyping method using Western blot analysis with specific antisera against three antigenically distinct OmlA proteins has been developed ${ }^{16,17}$. The Western blot analysis using specific antisera against $\mathrm{OmlA}_{5 \mathrm{a}(\mathrm{NG}-8)}$ (Fig. 1A), OmlA differentiate reference strains of $A$. pleuropneumoniae serotypes 1, 2, 4, 5a, 5b, 6, 7, 8, 9, 10, and 11 into three groups: Group A, serotypes 1, 2, 8, 9, and 11; Group B, serotypes 4, 6 and 7; Group C, serotypes 5a, 5b and 10 (Table 2). The reference strain of A. pleuropneumoniae serotype 3 and 12 did not express any detectable OmlA protein (Fig. 1 and Table 2). However, a field isolate of $A$. pleuropneumoniae serotype 3 could be grouped into Group B (Fig. 1B and Table 2). Regarding serotype 12 reference strain, Gerlach et al. ${ }^{11}$ observed a different result in Western blot analysis using a specific antiserum against the OmlA protein prepared from serotype 1 strain. They demonstrated that serum against the OmlA protein from a serotype 1 field isolate could detect an immuno- logically homologous protein in serotype 12 reference strain. An ORF (accession numbers: AB007574, AB007584) that has a sequence highly homologous to the $o m l A_{7}$ and oml $A_{1}$ gene is present in serotypes 3 and 12 reference strains stocked in our laboratory, respectively. Hence, serotypes 3 and 12 reference strains used in this study may not produce OmlA protein due to a genetic mutation in the region other than an ORF during repeated passages in laboratories. The three OmlA groups (groups A to C) by Western blot analysis could be distinguished by the nucleotide sequence-based typing (Table 2).

\section{Development of typing method based on the genetic diversity of the outer membrane lipoprotein $^{30}$}

A new genetic typing method using PCR-RFLP based on genetic diversity of the A. pleuropneumoniae omlA gene was developed ${ }^{30}$. Primers for PCR that amplify all A. pleuropneumoniae strains (5'-CCTAAGGTGATATGTCCGCA-3' and 5'-GTTGCCGTAGCACCGATTACG-3') were selected from a conserved sequence of the omlA of A. pleuropneumoniae. A DNA fragment of approximately 970 bp was amplified from the genomic DNA of 41 Japanese (serotypes 1, 2, 3, 5, and 7) and 1 Canadian (serotype 10) field isolates of $A$. pleuropneumoniae as well as that of reference strains of $A$. pleuropneumoniae serotypes 1 to 12 (data not shown), implying that the omlA gene is generally present in A. pleuropneumoniae field isolates as well as reference strains stocked in laboratories. Digestion of the DNA amplified from the reference and field strains with the enzymes VspI yielded specific polymorphic patterns, showing that the omlA genotype is inherent to a given serotype and allowing discrimination of all serotypes into five distinct groups: Group I, serotype 1, 9, 11, and 12; Group II, serotype 2 and 8; Group III, serotype 3, 6 and 7; Group IV, serotype 4; and Group V, serotype 5a, 5b and 10 (Fig. 3 and Table 2). Antigenic type of three OmlA protein groups by Western blot analysis (groups A to C) could be substituted by the PCR-RFLP typing since the antigenic groups could be predicted by the PCR-RFLP grouping (Table 2). OmlA serotyping with Western blot analysis may be the best typing method for examination of expression and antigenicity of the OmlA for vaccination. However, the PCRRFLP typing seems to be more useful for epidemiological study since the PCR-RFLP typing has more superior discriminatory ability than the Western blot analysis. Since the specific antisera for OmlA serotyping are not easily available in veterinary diagnostic laboratories, popularization of the OmlA serotyping may not be easy. On the contrary, the specific antisera are not required for 


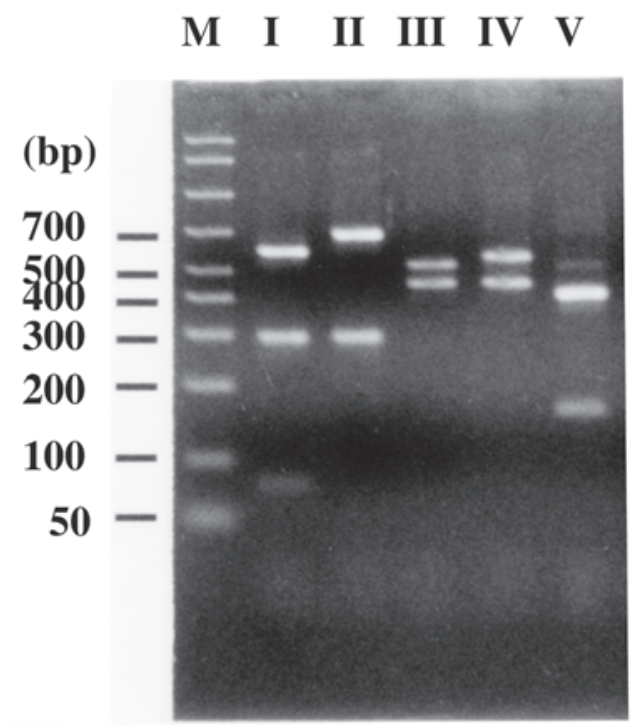

Fig. 3. The PCR-RFLP profiles of each groups after digestion with the restriction enzyme VspI

DNA size marker, $\mathrm{M}$, is shown on the left. Roman numerals above each lane correspond to the PCRRFLP group.

the PCR-RFLP typing and all reagents for the analysis are commercially available. Therefore, standard veterinary diagnostic laboratories equipped with PCR apparatus can easily do the OmlA typing by the PCR-RFLP typing method instead of OmlA serotyping by Western blot analysis.

To date, several PCR-RFLP-based or PCR-based typing methods using allelic variation of the target gene have been proposed for typing of A. pleuropneumoniae. The PCR-RFLP methods using the aroA gene encoding 5-enolpyruvylshikimate-3-phopshate synthase (a key enzyme in the biosynthesis of aromatic amino acids) ${ }^{15}$ and apxIV gene encoding an exotoxin (ApxIV) of A. pleuropneumoniae $^{18}$ have been developed for typing of the organism. However, it is unknown whether PCR tests for typing of the genes are protective antigens or not. Multiplex PCR tests for typing of the genes involved in the biosynthesis of capsular polysaccharides of A. pleuropneumoniae serotypes 2, 5 and $6^{19}$ and serotypes 1,2 and $8^{34}$ have also been developed. However, these tests cannot type $A$. pleuropneumoniae isolates other than serotypes $1,2,5,6$, and 8 .

Regarding typing methods whose target DNA is a gene for protective antigens of A. pleuropneumoniae other than the omlA gene, PCR assays based on the apx genes encoding toxins (ApxI, ApxII and ApxIII toxins) and a PCR-RFLP test based on the $t b p B$ gene encoding transferring-binding protein $(\mathrm{TbpB})$ which is a component of the iron-uptake system of $A$. pleuropneumoniae have been developed ${ }^{6,9,14,35}$. Multilocus typing of such genes encoding the protective antigens together with the omlA gene developed in this study would provide more effective and practical information on control and epidemiological analysis of porcine pleuropneumonia.

Consequently, the new genetic typing method of a protective OmlA protein developed in this study can be used as a routine tool which should be directly effective and practical for control and epidemiological analysis of porcine pleuropneumonia in veterinary diagnostic laboratories.

\section{Conclusion}

To examine antigenic type of A. pleuropneumoniae protective antigens is important for choice of vaccine. Therefore the genetic and antigenic typing methods of the OmlA protein described in this review used either alone or in combination should be directly effective and practical tools for control and epidemiological analysis of porcine pleuropneumonia caused by A. pleuropneumoniae.

\section{Acknowledgments}

The author gratefully thanks to Drs. Tsutomu Sekizaki, Makoto Osaki, Ikuo Uchida (National Institute of Animal Health, Japan), Nobuyuki Terakado (Kyoritsu Seiyaku, Japan) and Tadao Imada (National Institute of Animal Health, Japan) for their valuable guidance, advise, help and support for conducting the study in this review and writing this review.

\section{References}

1. Asawa, T. et al. (1995) Serotypes and antimicrobial susceptibility of Actinobacillus pleuropneumoniae isolated from piglets with pleuropneumonia. J. Vet. Med. Sci., 57, 757-759.

2. Babiuk, L. A. (2006) New applications for health and innovation in veterinary medicine. Adv. Pork Prod., 17, 169-179.

3. Blackall, P. J. et al. (2002) Proposal of a new serovar of Actinobacillus pleuropneumoniae: serovar 15. Vet. Microbiol., 84, 47-52.

4. Bunka, S. et al. (1995) Cloning and characterization of a protective outer membrane lipoprotein of Actinobacillus pleuropneumoniae serotype 5. Infect. Immun., 63, 27972800.

5. Cho, W. S. \& Chae, C. (2003) Differentiation of twelve Actinobacillus pleuropneumoniae serotypes by outer membrane lipoprotein gene-based restriction fragment length polymorphism. J. Vet. Med. B Infect. Dis. Vet. Public Health, 50, 90-94.

6. de la Puente-Redondo, V. A. et al. (2000) Detection and subtyping of Actinobacillus pleuropneumoniae strains by PCR-RFLP analysis of the $t b p A$ and $t b p B$ genes. Res. Microbiol., 151, 669-681.

7. Dubreuil, J. D. et al. (2000) Actinobacillus pleuropneu- 
moniae surface polysaccharides: their role in diagnosis and immunogenicity. Anim. Health Res. Rev., 1, 73-93.

8. Fittipaldi, N. et al. (2003) Evaluation and field validation of PCR tests for detection of Actinobacillus pleuropneumoniae in subclinically infected pigs. J. Clin. Microbiol., 41, 5085-5093.

9. Frey, J. et al. (1995) Development of an efficient PCR method for toxin typing of Actinobacillus pleuropneumoniae strains. Mol. Cell. Probes, 9, 277-282.

10. Fukuyasu, T., Saito, K. \& Ashida, K. (1996) Serotypes and antimicrobial susceptibility of Actinobacillus pleuropneumoniae isolated from pigs with pneumonia. Nihon juishikai zasshi (J. Jpn. Vet. Med. Assoc.), 49, 528-532 [In Japanese with English summary].

11. Gerlach, G. F. et al. (1993) Molecular characterization of a protective outer membrane lipoprotein (OmlA) from Actinobacillus pleuropneumoniae serotype 1 . Infect. Immun., 61, 565-572.

12. Gottschalk, M. et al. (2000) Atypical Actinobacillus pleuropneumoniae isolates that share antigenic determinants with both serotypes 1 and 7. J. Vet. Diagn. Invest., 12, 444-449.

13. Gram, T. \& Ahrens, P. (1998) Improved diagnostic PCR assay for Actinobacillus pleuropneumoniae based on the nucleotide sequence of an outer membrane lipoprotein. $J$. Clin. Microbiol., 36, 443-448.

14. Gram, T. et al. (2000) An Actinobacillus pleuropneumoniae PCR typing system based on the apx and omlA genes evaluation of isolates and tonsils of pigs. Vet. Microbiol., 75, 43-57.

15. Hernanz Moral, C. et al. (1999) Molecular cloning and sequencing of the aroA gene from Actinobacillus pleuropneumoniae and its use in a PCR assay for rapid identification. J. Clin. Microbiol., 37, 1575-1578.

16. Ito, H. et al. (1995) Molecular cloning of an Actinobacillus pleuropneumoniae outer membrane lipoprotein (OmlA) from serotype 5a. Microb. Pathog., 18, 29-36.

17. Ito, H. et al. (1998) Demonstration of the third antigenically distinct outer membrane lipoprotein (OmlA) in Actinobacillus pleuropneumoniae serotype 7. FEMS Microbiol. Lett., 167, 303-308.

18. Jaglic, Z. et al. (2004) Differentiation of Actinobacillus pleuropneumoniae by PCR-REA based on sequence variability of the apxIVA gene and by ribotyping. Vet. Microbiol., 103, 63-69.

19. Jessing, S. G., Angen, Ø. \& Inzana, T. J. (2003) Evaluation of a multiplex PCR test for simultaneous identification and serotyping of Actinobacillus pleuropneumoniae serotypes 2, 5, and 6. J. Clin. Microbiol., 41, 4095-4100.

20. Mittal, K. R., Higgins, R. \& Larivière, S. (1982) Evaluation of slide agglutination and ring precipitation tests for capsular serotyping of Haemophilus pleuropneumoniae. J. Clin. Microbiol., 15, 1019-1023.

21. Mittal, K. R., Higgins, R. \& Larivière, S. (1983) Identification and serotyping of Haemophilus pleuropneumoniae by coagglutination test. J. Clin. Microbiol., 18, 1351-1354.

22. Mittal, K. R., Higgins, R. \& Larivière, S. (1988) Quantitation of serotype-specific and cross-reacting group-specific antigens by coagglutination and immunodiffusion tests for differentiating Actinobacillus (Haemophilus) pleuropneumoniae strains belonging to cross-reacting serotypes 3, 6 and 8. J. Clin. Microbiol., 26, 985-989.
23. Mittal, K. R. (1990) Cross-reactions between Actinobacillus (Haemophilus) pleuropneumoniae strains of serotypes 1 and 9. J. Clin. Microbiol., 28, 535-539.

24. Mittal, K. R. \& Bourdon, S. (1991) Cross-reactivity and antigenic heterogeneity among Actinobacillus pleuropneumoniae strains of serotypes 4 and 7. J. Clin. Microbiol., 29, 1344-1347.

25. Mittal, K. R., Kamp, E. M. \& Kobisch, M. (1993) Serological characterization of Actinobacillus pleuropneumoniae strains of serotypes 1, 9 and 11. Res. Vet. Sci., 55, 179-184.

26. Nielsen, R. (1974) Serological and immunological studies of pleuropneumonia of swine caused by Haemophilus parahaemolyticus. Acta Vet. Scand., 15, 80-89.

27. Nielsen, R., \& O’Connor, P. J. (1984) Serological characterization of 8 Haemophilus pleuropneumoniae strains and proposal of a new serotype: serotype 8. Acta Vet. Scand., 25, 96-106.

28. Nielsen, R., Andresen, L. O. \& Plambeck, T. (1996) Serological characterization of Actinobacillus pleuropneumoniae biotype 1 strains antigenically related to both serotypes 2 and 7. Acta Vet. Scand., 37, 327-336.

29. Nielsen, R. et al. (1997) Serological characterization of Actinobacillus pleuropneumoniae biotype 2 strains isolated from pigs in two Danish herds. Vet. Microbiol., 54, 35-46.

30. Osaki, M. et al. (1997) Genetic diversity of the genes encoding the outer membrane lipoprotein (omlA) of Actinobacillus pleuropneumoniae. J. Vet. Med. Sci., 59, 213-215.

31. Perry, M. B. et al. (1990) Structural characteristics of the antigenic capsular polysaccharides and lipopolysaccharides involved in the serological classification of Actinobacillus (Haemophilus) pleuropneumoniae strains. Serodiag. Immunother. Infect. Dis., 4, 299-308.

32. Rigout, S. \& Chevallier, B. (1999) Development and advantage of a multiplex PCR assay for the detection and typing of Actinobacillus pleuropneumoniae. J. Rech. Porcine en France, 31, 365-370 [In French with English summary].

33. Savoye, C. et al. (2000) A PCR assay used to study aerosol transmission of Actinobacillus pleuropneumoniae from samples of live pigs under experimental conditions. Vet. Microbiol., 73, 337-347.

34. Schuchert, J. A. et al. (2004) Detection and identification of Actinobacillus pleuropneumoniae serotypes 1, 2, and 8 by multiplex PCR. J. Clin. Microbiol., 42, 4344-4348.

35. Sthimatee, N. et al. (2003) Identification of the Actinobacillus pleuropneumoniae serotype using PCR-based-apx genes. Mol. Cell. Probes, 17, 301-305.

36. Taylor, D. J. (1999) Actinobacillus pleuropneumoniae. In Disease of swine 8th edition, eds. Straw, B. E. et al., Iowa State University Press, Ames, U. S. A., 343-354.

37. Tezuka, K. \& Suzuki, T. (2002) Case report: an outbreak of porcine pleuropneumoniae in a pig farm. Rinsho Juui (J. Clin. Vet. Med.), 20, 52-56 [In Japanese].

38. Tonpitak, W., Rohde, J. \& Gerlach, G. F. (2007) Prevalence of "Actinobacillus porcitonsillarum" in porcine tonsils and development of a diagnosis duplex PCR differentiating between "Actinobacillus porcitonsillarum" and Actinobacillus pleuropneumoniae. Vet. Microbiol., 122, 157-165.

39. Yoshimura, H. et al. (2002) Comparative in vitro activity of 16 antimicrobial agents against Actinobacillus pleuropneumoniae. Vet. Res. Commun., 26, 11-19. 